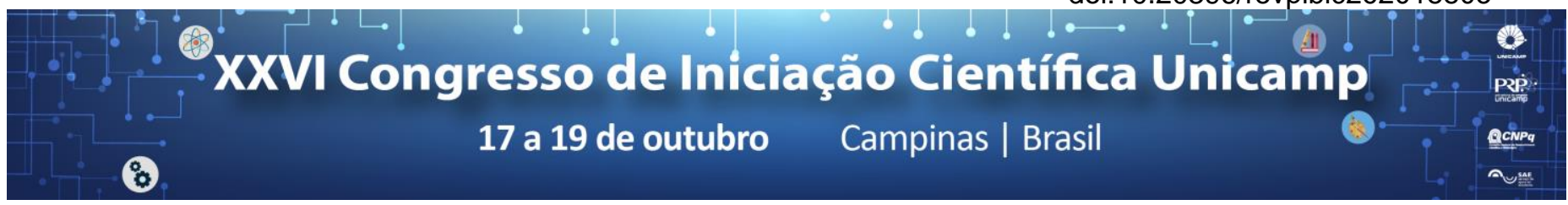

\title{
Anéis Noetherianos
}

\section{Leandro P. Guidio*, Ronan Antonio dos Reis}

\section{Resumo}

Este trabalho trata de um estudo introdutório em Teoria de Anéis e Ideais, em que foram estudados conceitos, propriedades e resultados envolvendo anéis, ideais, em especial, a classe dos anéis Noetherianos, ou seja, anéis que satisfazem uma certa condição de cadeia ascendente para ideais. O objetivo principal deste trabalho é apresentar um estudo sobre o conhecido Teorema da Base de Hilbert, ou seja, que todo anel de polinômios em uma variável com coeficientes num anel Noetheriano é um anel Noetheriano, bem como, que esse resultado também é válido para anel de polinômios em várias variáveis. Este estudo aparece em várias áreas, tais como, em Geometria Algébrica, Álgebra comutativa, entre outras.

\section{Palavras-chave:}

Anéis e Ideais, Anéis Noetherianos, Teorema da Base de Hilbert.

\section{Introdução}

A Teoria de Anéis e Ideais é uma área de grande importância em várias áreas da ciência, tais como, em Álgebra, Geometria Algébrica, Álgebra comutativa. Uma classe de anéis que aparecem e desempenham um papel importante nesta Teoria é a dos anéis Noetherianos, ou seja, anéis que satisfazem certa condição de cadeia ascendente para ideais. $O$ objetivo principal deste trabalho é apresentar um estudo sobre o conhecido Teorema da Base de Hilbert, ou seja, que todo anel de polinômios em uma variável com coeficientes num anel Noetheriano é um anel Noetheriano, bem como, que esse resultado também é válido para anel de polinômios em várias variáveis. O termo Noetheriano é uma homenagem à matemática alemã Emmy Noether. Este trabalho foi feito com base nas referências bibliográficas.

\section{Resultados e Discussão}

Seja A um anel com as operações de adição + e multiplicação . .

Iniciemos com o seguinte conceito:

Definição 1: Um anel A é dito comutativo se $a b=b a$, $a, b \in A$.

Definição 2: Um subconjunto não vazio I do anel comutativo $\mathrm{A}$, é dito Ideal de $\mathrm{A}$, se satisfaz as propriedades:

i) Dados quaisquer $\mathrm{x}, \mathrm{y} \in \mathrm{I}$ temos $\mathrm{x}-\mathrm{y} \in \mathrm{I}$;

ii) Dados quais $a \in A$ e $x \in I$, então a. $x \in I$.

Definição 3: Um anel A satisfaz a condição de cadeia ascendente para ideais se dada uma sequência de ideais $\mathrm{I}_{1}, \mathrm{I}_{2}, \ldots, \mathrm{I}_{\mathrm{n}}, \ldots$ de $\mathrm{A}$ com $\mathrm{I}_{1} \subseteq \mathrm{I}_{2} \subseteq \ldots \subseteq \mathrm{I}_{\mathrm{n}} \ldots$.

existe $u m$ inteiro $\mathrm{n}$ tal que $\mathrm{I}_{\mathrm{m}}=\mathrm{I}_{\mathrm{n}}$ para todo $\mathrm{m} \geq \mathrm{n}$.

Definição 4: A condição de máximo (para ideais) é satisfeita em A se cada conjunto não vazio de ideais em A, parcialmente ordenado pela inclusão possui um elemento maximal.
Definição 5: Um Ideal I é dito finitamente gerado, se existem $a_{1}, a_{2}, . ., a_{n}$ em $A$ tais que qualquer elemento de I pode ser escrito da forma $x_{1} a_{1}+x_{2} a_{2}+. .+x_{n} a_{n}$ com $x_{1}$, $\mathrm{x}_{2}, . ., \mathrm{x}_{\mathrm{n}} \in \mathrm{A}$.

Teorema 1: São equivalentes as afirmações abaixo sobre ideais de um anel A:

i) A satisfaz a condição de cadeia ascendente para ideais.

ii) A condição de máximo é satisfeita em $\mathrm{A}$.

iii) Cada ideal de A é finitamente gerado.

Definição 6: Um anel que satisfaz uma das três condições do Teorema 1 é dito Anel Noetheriano.

Exemplo 1: $O$ anel $\mathbb{Z}$ dos inteiros, o anel $\mathbb{R}[\mathrm{x}]$ com coeficientes no corpo dos números reais $(\mathbb{R})$ com as respectivas operações usuais de adição e multiplicação é um anel Noetheriano.

O seguinte resultado é conhecido como Teorema da base de Hilbert:

Teorema 2: Se um anel A é Noetheriano, então o anel de polinômios $\mathrm{A}[\mathrm{x}]$ é também Noetheriano.

Corolário 1: Se um anel A é Noetheriano, então o anel de polinômios $\mathrm{A}\left[\mathrm{x}_{1}, \ldots, \mathrm{x}_{\mathrm{n}}\right]$ é Noetheriano.

\section{Conclusões}

A partir do estudo de tópicos da Teoria de Anéis e Ideais, estudamos a classe dos Anéis Noetherianos, em que mostramos alguns resultados, tais como, o Teorema da Base de Hilbert, e consequência.

BURTON, D.M. A first Course in Rings and Ideals. Addison-Wesley Publishing Company, 1970.

${ }^{2}$ FRALEIGH, J.B. A First Course in Abstract Algebra. Addison-Wesley Publishing Company, 1993.

${ }^{3}$ GARCIA, A. e LEQUAIN, Y. Álgebra: Um Curso de Introdução. IMPA, 1988.

${ }^{4}$ GONÇALVES, A. Introdução à Álgebra. IMPA, 1979

${ }^{5}$ HERSTEIN, I.N. Tópicos de Álgebra. São Paulo: Editora Polígono, 1970. 